\title{
Antiviral Peptide as Promising Therapeutics against SARS-CoV-2
}

Surid Mohammad Chowdhury ${ }^{1,2}$, Shafi Ahmad Talukder ${ }^{1}$, Akib Mahmud Khan ${ }^{1,3}$, Nadia Afrin ${ }^{1}$, Md Ackas Ali ${ }^{1}$, Rajib Islam ${ }^{1}$, Rimon Parves $^{1}$, Abdulla Al Mamun ${ }^{4}$, Md. Abu Sufian ${ }^{5}$, Md Nayeem Hossain ${ }^{1}$, Mohammed Akhter Hossain ${ }^{6}$, Mohammad A. Halim,7*

${ }^{1}$ Division of Infectious Diseases and Division of Computer-Aided Drug Design, The Red-Green Research Centre, BICCB, 16 Tejkunipara, Tejgaon, Dhaka, 1215, Bangladesh

${ }^{2}$ Department of Pharmacy, Southeast University, Dhaka 1213, Bangladesh

${ }^{3}$ Department of Oncological Sciences, University of Utah, Salt Lake City, Utah 84418, USA

${ }^{4}$ Key Laboratory of Soft Chemistry and Functional Materials of MOE, School of Chemical Engineering, Nanjing University of Science and Technology, Nanjing 210094, China

${ }^{5}$ School of Pharmacy, Temple University, Philadelphia, Pennsylvania, 19122, USA

${ }^{6}$ Florey Institute of Neuroscience and Mental Health, University of Melbourne, Melbourne, VIC 3010, Australia

${ }^{7}$ Department of Physical Sciences, University of Arkansas-Fort Smith, Fort Smith, Arkansas 72913, USA

*Correspondence: mohammad.halim@uafs.edu 
Table S1. Sequence, length, inhibition efficiency of the 51 antiviral peptides against different cell lines infected by SARS-CoV-1.

\begin{tabular}{|c|c|c|c|c|c|}
\hline Peptide ID & Sequence & Length & Cell_Line & Inhibition/IC50 & Unit \\
\hline S2P1 & MWKTPTLKYFGGFNFSQI & 18 & Vero E6 & 58 & $\%$ \\
\hline S2P2 & ATAGWTFGAGAALQIPFAMQMAY & 23 & Vero E6 & 39 & $\%$ \\
\hline S2P3 & GYHLMSFPQAAPHGVVFLHVTW & 22 & Vero E6 & 2 & $\mu \mathrm{M}$ \\
\hline S2P4 & GVFVFNGTSWFITQRNFFS & 19 & Vero E6 & 2 & $\mu \mathrm{M}$ \\
\hline S2P5 & NEVAKNLNESLIDLQELGKYEQYIKWPWYVW & 31 & Vero E6 & Nil & - \\
\hline S2P6 & AACEVAKNLNESLIDLQELGKYEQYIKW & 28 & Vero E6 & 42 & $\%$ \\
\hline S2P7 & FKLPLGINITNFRAILTAFS & 20 & FRhK-4 & $>500$ & $\begin{array}{l}\text { IC90 } \\
(\mu \mathrm{g} / \mathrm{ml})\end{array}$ \\
\hline S2P8 & PTTFMLKYDENGTITDAVDC & 20 & FRhK-4 & $112.5 \pm 26.3$ & $\begin{array}{l}\text { IC90 } \\
(\mu \mathrm{g} / \mathrm{ml})\end{array}$ \\
\hline S2P9 & VLYNSTFFSTFKCYGVSATK & 20 & FRhK-4 & $>500$ & $\begin{array}{l}\mathrm{IC} 90 \\
(\mu \mathrm{g} / \mathrm{ml})\end{array}$ \\
\hline S2P10 & PALNCYWPLNDYGFYTTSGI & 20 & FRhK-4 & $>500$ & $\begin{array}{l}\mathrm{IC} 90 \\
(\mu \mathrm{g} / \mathrm{ml})\end{array}$ \\
\hline S2P11 & RDVSDFTDSVRDPKTSEILD & 20 & FRhK-4 & $>500$ & $\begin{array}{l}\text { IC90 } \\
(\mu \mathrm{g} / \mathrm{ml})\end{array}$ \\
\hline S2P12 & YQDVNCTDVSTAIHADQLTP & 20 & FRhK-4 & $113.0 \pm 27.6$ & $\begin{array}{l}\text { IC90 } \\
(\mu \mathrm{g} / \mathrm{ml})\end{array}$ \\
\hline S2P13 & SNNTIAIPTNFSISITTEVM & 20 & FRhK-4 & $>500$ & $\begin{array}{l}\text { IC90 } \\
(\mu \mathrm{g} / \mathrm{ml})\end{array}$ \\
\hline S2P14 & QYGSFCTQLNRALSGIAAEQ & 20 & FRhK-4 & $24.9 \pm 6.2$ & $\begin{array}{l}\text { IC90 } \\
(\mu \mathrm{g} / \mathrm{ml})\end{array}$ \\
\hline S2P15 & GIGVTQNVLYENQKQIANQF & 20 & FRhK-4 & $>500$ & $\begin{array}{l}\text { IC90 } \\
(\mu \mathrm{g} / \mathrm{ml})\end{array}$ \\
\hline S2P16 & IQKEIDRLNEVAKNLNESLI & 20 & FRhK-4 & $73.5 \pm 15.7$ & $\begin{array}{l}\text { IC90 } \\
(\mu \mathrm{g} / \mathrm{ml})\end{array}$ \\
\hline S2P17 & GINASVVNIQKEIDRLNEVAKNLNESLIDL & 30 & $\mathrm{HeLa}$ & 0.8 & $\mu \mathrm{M}$ \\
\hline S2P18 & GINASVVNIQKEIDRLNEVAKNL & 23 & $\mathrm{HeLa}$ & 1.04 & $\mu \mathrm{M}$ \\
\hline
\end{tabular}




\begin{tabular}{|c|c|c|c|c|c|}
\hline S2P19 & ISGINASVVNIQEEIKKLNEEAKKLNESLIDLQEL & 35 & Vero E6 & 4 & $\mathrm{nM}$ \\
\hline S2P20 & ISGINASVVNIQKEIDRLNEVAKNLNESLIDLQEL & 35 & Vero E6 & 5 & $\mathrm{nM}$ \\
\hline S2P21 & IEEINKKVEEIQKKIEELNKKAEELNKKLEELQKK & 35 & Vero E6 & $>100$ & $\mu \mathrm{M}$ \\
\hline S2P22 & ISGINASVVNIQKEIDRLNEVAKNLNESLIDLQEL & 35 & Vero E3 & 0.34 & $\mu \mathrm{M}(\mathrm{EC} 50)$ \\
\hline S2P23 & YENQKQIANQFNKAISQIQESLTTTSTA & 28 & Vero E3 & 1.16 & $\mu \mathrm{M}(\mathrm{EC} 50)$ \\
\hline S2P24 & IQESLTTTSTALGKLQDVVNQNAQALNTLVKQLSS & 35 & Vero E3 & ND & $\mu \mathrm{M}(\mathrm{EC} 50)$ \\
\hline S2P25 & FGGASCCLYCRCHIDHPNPKGFCDLKGKY & 29 & E. coli & 160 & $\mu \mathrm{M}$ \\
\hline S2P26 & ALNCYWPLNDYGFYTTTGIGYQPYRVVVLSFEL & 33 & Vero & 41.6 & $\mu \mathrm{M}$ \\
\hline S2P27 & CANLLLQYGSFCTQLNRALSGIA & 23 & HEK293T & 50 & $\%$ \\
\hline S2P28 & PSSKRFQPFQQFGRDVSDFT & 20 & HEK293T & 50 & $\%$ \\
\hline S2P29 & RNTREVFAQVKQMYKTPTLKYFG & 23 & HEK293T & Low & - \\
\hline S2P30 & TKFPSVYAWERKKISNCVAD & 20 & HEK293T & Low & - \\
\hline S2P31 & KGIYQTSNFRVVPSGDVVRF & 20 & HEK293T & Nil & - \\
\hline S2P32 & KSNVVRGWVFGSTMNNKSQS & 20 & HEK293T & Nil & - \\
\hline S2P33 & TDAVDCSQNPLAELKCSVKSF & 21 & HEK293T & Nil & - \\
\hline S2P34 & TSSMRGVYYPDEIFRSDTLYL & 21 & HEK293T & Nil & - \\
\hline S2P35 & YKGYQPIDVVRDLPSGFNTL & 20 & HEK293T & Nil & - \\
\hline S2P36 & IQKEIDRLNEVAKNLNESLIDLQELGK & 27 & $293 \mathrm{~T}$ & 1.19 & $\mu \mathrm{M}(\mathrm{EC} 50)$ \\
\hline S2P37 & ISGINASVVNIQKEIDRLNEVAKNLNESLIDLQEL & 35 & $293 \mathrm{~T}$ & Low & NA \\
\hline S2P38 & GINASVVNIQKEIDRLNEVAKNLNESLIDLQELGK & 35 & $293 \mathrm{~T}$ & Low & NA \\
\hline S2P39 & INASVVNIQKEIDRLNEVAKNLNESLIDLQELGK & 34 & $293 \mathrm{~T}$ & Low & NA \\
\hline $\mathrm{S} 2 \mathrm{P} 40$ & INASVVNIQKEIDRLNEVAKNLNESLIDL & 29 & $293 \mathrm{~T}$ & Low & NA \\
\hline S2P41 & VVNIQKEIDRLNEVAKNLNESLIDLQELGK & 30 & $293 \mathrm{~T}$ & Low & NA \\
\hline $\mathrm{S} 2 \mathrm{P} 42$ & IDRLNEVAKNLNESLIDLQELGKYEQYIKWPW & 32 & $293 \mathrm{~T}$ & Low & NA \\
\hline $\mathrm{S} 2 \mathrm{P} 43$ & DLSLDFEKLNVTLLDLTYEMNRIQDAIKKLNESYINLKE & 39 & Vero & $>50$ & $\mu \mathrm{M}(\mathrm{EC} 50)$ \\
\hline $\mathrm{S} 2 \mathrm{P} 44$ & GVTQNVLYENQKQIANQFNKAISQIQESLTTTSTALGKLQ & 40 & Vero E6 & Low & NA \\
\hline $\mathrm{S} 2 \mathrm{P} 45$ & LTTTSTALGKLQDVVNQNAQALNTLVKQLSSNFG & 34 & Vero E6 & No & NA \\
\hline S2P46 & KQLSSNFGAISSVLNDILSRLDKVEAEVQIDRLITG & 36 & Vero E6 & No & NA \\
\hline S2P47 & GRLQSLQTYVTQQLIRAAEIRASANLAATKMSEC & 34 & Vero E6 & No & NA \\
\hline $\mathrm{S} 2 \mathrm{P} 48$ & GINASVVNIQKEIDRLNEVAKNLNESLIDLQELGKYE & 37 & Vero E6 & 19 & $\mu \mathrm{mol} / 1$ \\
\hline
\end{tabular}




\begin{tabular}{|l|l|l|l|l|l|}
\hline S2P49 & KEIDRLNEVAKNLNESLIDLQELGKYEQYIKWPWYVW & 37 & Vero E6 & No & NA \\
\hline S2P50 & LFGLIPSLIGGLVSAFK & 17 & MDCK & 7.12 & $\mu$ (EC50) \\
\hline S2P51 & LFRLIKSLIKRLVSAFK & 17 & MDCK & No & NA \\
\hline $\begin{array}{l}\alpha 1 \text { helix of } \\
\text { peptidase } \\
\text { domain of } \\
\text { RBD }\end{array}$ & TIEEQAKTFLDKFNHEAEDLFYQSSLASWNYNT & 33 & & & \\
\hline
\end{tabular}


Table S2. Binding affinity and score of peptides against the RBD of SARS CoV-2 spike protein obtained from Firedock, ClusPro and HADDOCK.

\begin{tabular}{|l|c|c|c|c|}
\hline Peptide ID & Length & Firedock_score & Cluspro_score & Haddock score \\
\hline S2P1 & 18 & -40.02 & -716 & $-111.5+/-1.6$ \\
\hline S2P3 & 22 & -43.94 & -757.6 & $-94.7+/-4.1$ \\
\hline S2P4 & 19 & -35.09 & -710.1 & $-95.6+/-7.0$ \\
\hline S2P5 & 31 & -34.94 & -844.2 & $-95.0+/-4.1$ \\
\hline S2P6 & 28 & -28.94 & -613.7 & $-82.4+/-7.8$ \\
\hline S2P7 & 20 & -31.62 & -682.4 & $-59.3+/-3.6$ \\
\hline S2P8 & 20 & -29.88 & -519 & $-88.8+/-4.8$ \\
\hline S2P9 & 20 & -35.76 & -704.6 & $-102.0+/-2.1$ \\
\hline S2P10 & 20 & -40.92 & -892.8 & $-95.3+/-2.4$ \\
\hline S2P11 & 20 & -18.36 & -494 & $-82.6+/-3.3$ \\
\hline S2P12 & 20 & -29.6 & -527.7 & $-82.3+/-1.0$ \\
\hline S2P13 & 20 & -24.73 & -642.1 & $-68.7+/-1.8$ \\
\hline S2P14 & 20 & -27.91 & -650.1 & $-93.8+/-10.3$ \\
\hline S2P15 & 20 & -37.45 & -635.3 & $-87.3+/-4.5$ \\
\hline S2P16 & 20 & -26.97 & -555.5 & $-71.9+/-1.1$ \\
\hline S2P17 & 30 & -36.09 & -567.9 & $-82.5+/-3.1$ \\
\hline S2P18 & 23 & -21.29 & -556.9 & $-83.1+/-3.4$ \\
\hline S2P19 & 35 & -38.32 & -641.5 & $-73.7+/-1.0$ \\
\hline S2P20 & 35 & -24 & -580.8 & $-83.4+/-3.7$ \\
\hline S2P21 & 35 & -36.15 & -563.4 & $-70.0+/-8.9$ \\
\hline S2P22 & 35 & -40.24 & -673.5 & $-70.0+/-6.4$ \\
\hline S2P23 & 28 & -28.09 & -624.9 & $-97.9+/-1.7$ \\
\hline S2P24 & 35 & -42.69 & -632.5 & $-77.1+/-2.7$ \\
\hline S2P25 & 29 & -44.17 & -742.4 & $-112.4+/-3.3$ \\
\hline S2P26 & 23 & -63.1 & -744.5 & $-105.0+/-1.9$ \\
\hline S2P27 & -36.81 & -699.7 & $-90.5+/-2.5$ \\
\hline
\end{tabular}




\begin{tabular}{|l|l|l|l|l|}
\hline S2P28 & 20 & -36.41 & -677.8 & $-108.1+/-3.8$ \\
\hline S2P29 & 23 & -28.66 & -632.5 & $-94.7+/-4.0$ \\
\hline S2P30 & 20 & -29.56 & -652.8 & $-109.7+/-3.9$ \\
\hline S2P31 & 20 & -32.71 & -679.8 & $-87.2+/-1.6$ \\
\hline S2P32 & 20 & -31.81 & -650.4 & $-80.6+/-3.6$ \\
\hline S2P33 & 21 & -30.99 & -598.9 & $-76.6+/-4.7$ \\
\hline S2P34 & 21 & -36.86 & -642.3 & $-102.8+/-0.7$ \\
\hline S2P35 & 20 & -29.22 & -805.4 & $-86.9+/-4.7$ \\
\hline S2P36 & 27 & -27.18 & -568.4 & $-78.0+/-2.9$ \\
\hline S2P37 & 35 & -27.02 & -617.5 & $-79.7+/-2.9$ \\
\hline S2P38 & 35 & -37.7 & -661.4 & $-71.2+/-5.3$ \\
\hline S2P39 & 34 & -26.02 & -611 & $-84.6+/-1.6$ \\
\hline S2P40 & 29 & -23 & -618.3 & $-71.5+/-1.7$ \\
\hline S2P41 & 30 & -34.72 & -685.5 & $-72.7+/-7.5$ \\
\hline S2P42 & 32 & -40.01 & -747.9 & $-99.2+/-7.0$ \\
\hline S2P43 & 39 & -34.72 & -644.8 & $-102.9+/-1.8$ \\
\hline S2P44 & 40 & -31.48 & -595.3 & $-89.4+/-3.5$ \\
\hline S2P45 & 34 & -33.75 & -657.3 & $-71.3+/-1.7$ \\
\hline S2P46 & 36 & -37.79 & -751.7 & $-77.0+/-4.0$ \\
\hline S2P47 & 34 & -29.51 & -647.7 & $-81.5+/-2.6$ \\
\hline S2P48 & 37 & -33.98 & -657.7 & $-84.7+/-2.8$ \\
\hline S2P49 & 37 & -49.97 & -846.8 & $-96.2+/-3.1$ \\
\hline S2P50 & 17 & -34.33 & -628.2 & $-88.7+/-1.2$ \\
\hline S2P51 & -23 & -36.74 & -644.1 & $-84.7+/-9.1$ \\
\hline$\alpha 1$ helix of peptidase domain of RBD & -23.37 & -749.6 & $-118.2+/-1.7$ \\
\hline MIT peptide & -588.3 & $-95.2+/-2.2$ \\
\hline
\end{tabular}


Table S3. Stepwise multiple linear regression analysis with most relevant peptide properties as predictors of binding affinity (Firedock global energy) of top 15 peptides with the RBD of SARS CoV-2 spike protein.

\begin{tabular}{|c|c|c|c|c|c|c|}
\hline & $\begin{array}{c}\text { Step 1 } \\
\left(\mathrm{R}^{2}=0.928\right)\end{array}$ & $\begin{array}{c}\text { Step 2 } \\
\left(\mathrm{R}^{2}=0.924\right)\end{array}$ & $\begin{array}{c}\text { Step 3 } \\
\left(\mathrm{R}^{2}=0.924\right.\end{array}$ & $\begin{array}{c}\text { Step 4 } \\
\left(\mathrm{R}^{2}=0.907\right.\end{array}$ & $\begin{array}{c}\text { Step 5 } \\
\left(\mathrm{R}^{2}=0.906\right)\end{array}$ & $\begin{array}{c}\text { Step 6 } \\
\left(\mathrm{R}^{2}=0.894\right)\end{array}$ \\
\hline $\begin{array}{c}\text { Predictors of } \\
\text { binding affinity }\end{array}$ & \multicolumn{7}{|c|}{ P Values } \\
\hline Aromatic AA & 0.197 & 0.185 & $1.85 \times 10^{-5}$ & $1.24 \times 10^{-5}$ & $4.13 \times 10^{-6}$ & $1.63 \times 10^{-6}$ \\
\hline Nonpolar AA & 0.581 & 0.572 & 0.049 & 0.004 & 0.002 & 0.000 \\
\hline Polar AA & 0.508 & 0.523 & 0.024 & 0.036 & 0.029 & 0.011 \\
\hline Acidic AA (-ve) & 0.417 & 0.379 & 0.140 & 0.324 & $\mathbf{0 . 2 7 1}$ & \\
\hline Net charge at PH 7 & 0.254 & 0.243 & 0.214 & $\mathbf{0 . 7 3 7}$ & & \\
\hline Basic AA (+ve) & 0.416 & 0.445 & $\mathbf{0 . 2 2 3}$ & & & \\
\hline MW & 0.616 & $\mathbf{0 . 8 7 6}$ & & & & \\
\hline Vol (A $\left.\mathrm{A}^{3}\right)$ & $\mathbf{0 . 6 1 7}$ & & & & \\
\hline
\end{tabular}


Table S4: 10 high frequency residues and their types in the contact maps of top 3 peptides with RBD of SARS CoV-2 spike protein

\begin{tabular}{|c|c|c|c|c|c|c|c|c|c|c|}
\hline & \multicolumn{10}{|c|}{10 High frequency residues (highest to lowest) } \\
\hline AH & Glu 18 & His 15 & Gln 23 & Lys 12 & Gln 5 & Tyr 22 & Asp 11 & Asp 19 & Glu 16 & Asn 30 \\
\hline AA type & A & B & P & NP & P & AR & A & A & A & P \\
\hline S2P25 & Tyr 29 & Cys 10 & His 13 & Cys 12 & Phe 1 & Arg 11 & Gly 3 & Gly 2 & Phe 22 & Ala 4 \\
\hline AA type & AR & P & B & P & AR & B & NP & NP & AR & NP \\
\hline S2P26 & Pro 7 & Tyr 21 & Cys 4 & Tyr 5 & Tyr 24 & Trp 6 & Gly 20 & Asn 3 & Phe 31 & Leu 8 \\
\hline AA type & NP & AR & P & AR & AR & AR & NP & P & AR & NP \\
\hline
\end{tabular}

* $\mathrm{A}=$ acidic/AA with negative side chain, $\mathrm{B}=$ basic/AA with positive side chain, $\mathrm{P}=$ polar $\mathrm{AA}, \mathrm{NP}=$ nonpolar $\mathrm{AA}, \mathrm{AR}=$ aromatic AA 
Table S5: Peptides binding affinities and physicochemical properties used in structure activity relationship analyses

\begin{tabular}{|l|l|l|l|l|l|l|l|l|l|}
\hline $\begin{array}{l}\text { Peptide } \\
\text { ID }\end{array}$ & $\begin{array}{l}\text { Binding affinity } \\
\text { (Firedock global } \\
\text { energy) }\end{array}$ & $\begin{array}{l}\text { Aromatic } \\
\text { AA }\end{array}$ & $\begin{array}{l}\text { Non-polar } \\
\text { AA }\end{array}$ & $\begin{array}{l}\text { Polar } \\
\text { AA }\end{array}$ & $\begin{array}{l}\text { Acidic } \\
\text { AA }\end{array}$ & $\begin{array}{l}\text { Basic } \\
\text { AA }\end{array}$ & $\begin{array}{l}\text { Net } \\
\text { charge at } \\
\text { pH 7 }\end{array}$ & $\begin{array}{l}\text { MW } \\
\text { volume (A3) }\end{array}$ \\
\hline S2P1 & -40.02 & 5 & 6 & 5 & 0 & 2 & 2 & 2165.54 & 2621 \\
\hline S2P3 & -43.94 & 4 & 12 & 3 & 0 & 3 & 0.3 & 2494.9 & 3019 \\
\hline S2P10 & -40.92 & 5 & 8 & 6 & 1 & 0 & -1.1 & 2295.55 & 2778 \\
\hline S2P15 & -37.45 & 2 & 8 & 8 & 1 & 1 & 0 & 2264.52 & 2739 \\
\hline S2P19 & -38.32 & 0 & 15 & 9 & 7 & 4 & -3 & 3923.47 & 4747 \\
\hline S2P22 & -40.24 & 0 & 16 & 10 & 6 & 3 & -3 & 3893.41 & 4711 \\
\hline S2P24 & -42.69 & 0 & 14 & 17 & 2 & 2 & 0 & 3715.17 & 4495 \\
\hline S2P25 & -44.17 & 4 & 10 & 7 & 2 & 6 & 1.9 & 3233.76 & 3913 \\
\hline S2P26 & -63.1 & 8 & 14 & 8 & 2 & 1 & -1.1 & 3864.39 & 4675 \\
\hline S2P27 & -36.81 & 2 & 11 & 9 & 0 & 1 & 0.9 & 2456.86 & 2973 \\
\hline S2P34 & -36.86 & 4 & 7 & 5 & 3 & 2 & -1 & 2513.8 & 3042 \\
\hline S2P38 & -37.7 & 0 & 16 & 9 & 6 & 4 & -2 & 3878.39 & 4692 \\
\hline S2P42 & -40.01 & 4 & 12 & 6 & 6 & 4 & -2 & 3919.45 & 4742 \\
\hline S2P46 & -37.79 & 1 & 16 & 10 & 5 & 4 & -1 & 3930.47 & 4755 \\
\hline S2P49 & -49.97 & 6 & 13 & 6 & 7 & 5 & -2 & 4625.26 & 5596 \\
\hline AH & -36.74 & 6 & 7 & 12 & 6 & 3 & -3.9 & 4055.34 & 4907 \\
\hline
\end{tabular}



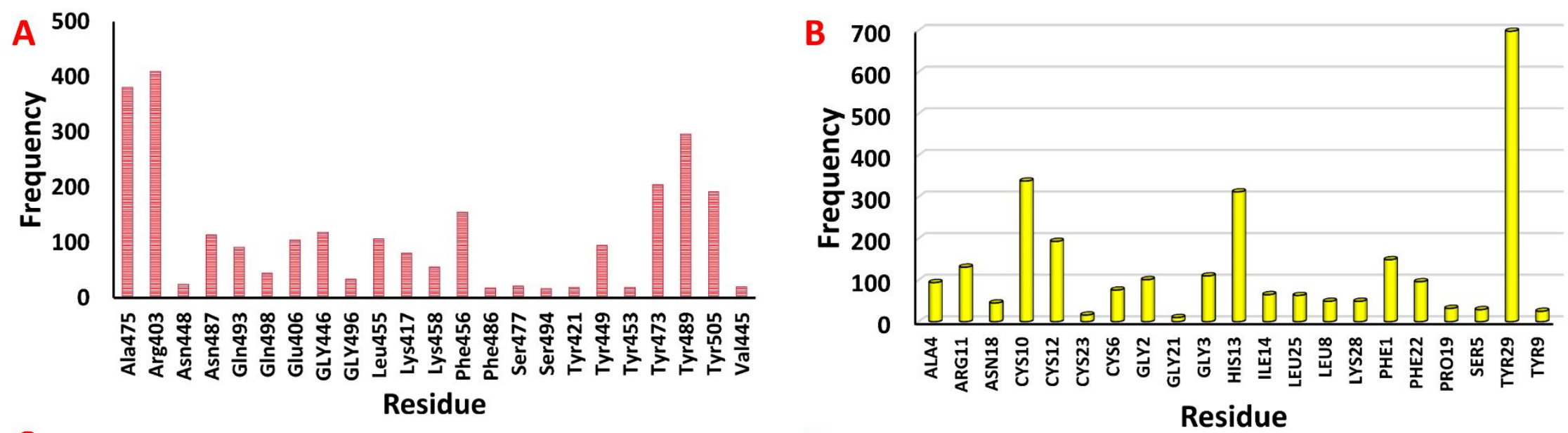

C

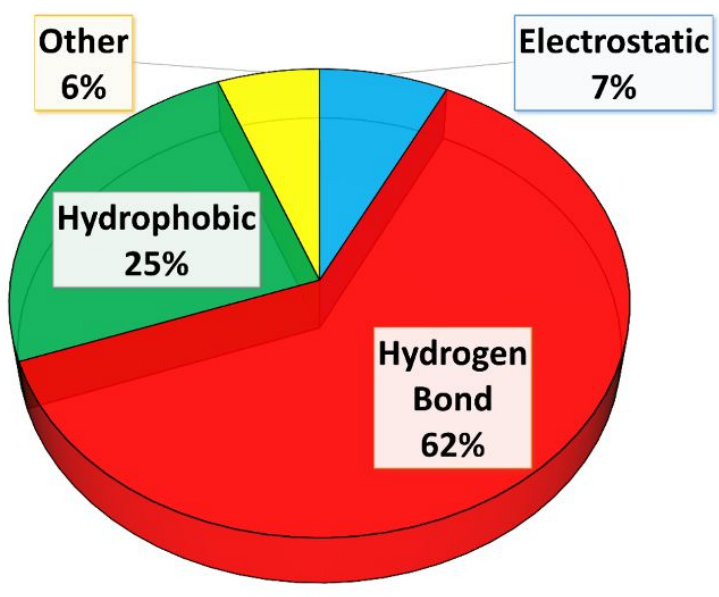

D

\section{Residue}

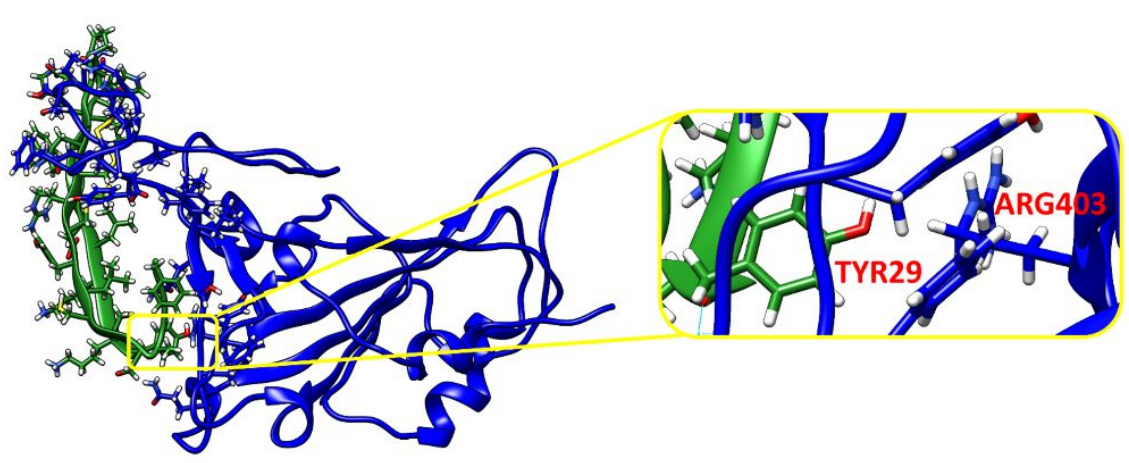

Figure S1. (a) Interacting residues in RBD; (b) Interacting residues in peptide S2P25; (c) Distribution of non-covalent interactions in S2P25-RBD complex obtained from 150 ns simulation time span; (d) A representative snapshot of S2P25-RBD complex highlighting interaction between S2P25 (green) and RBD (blue). 

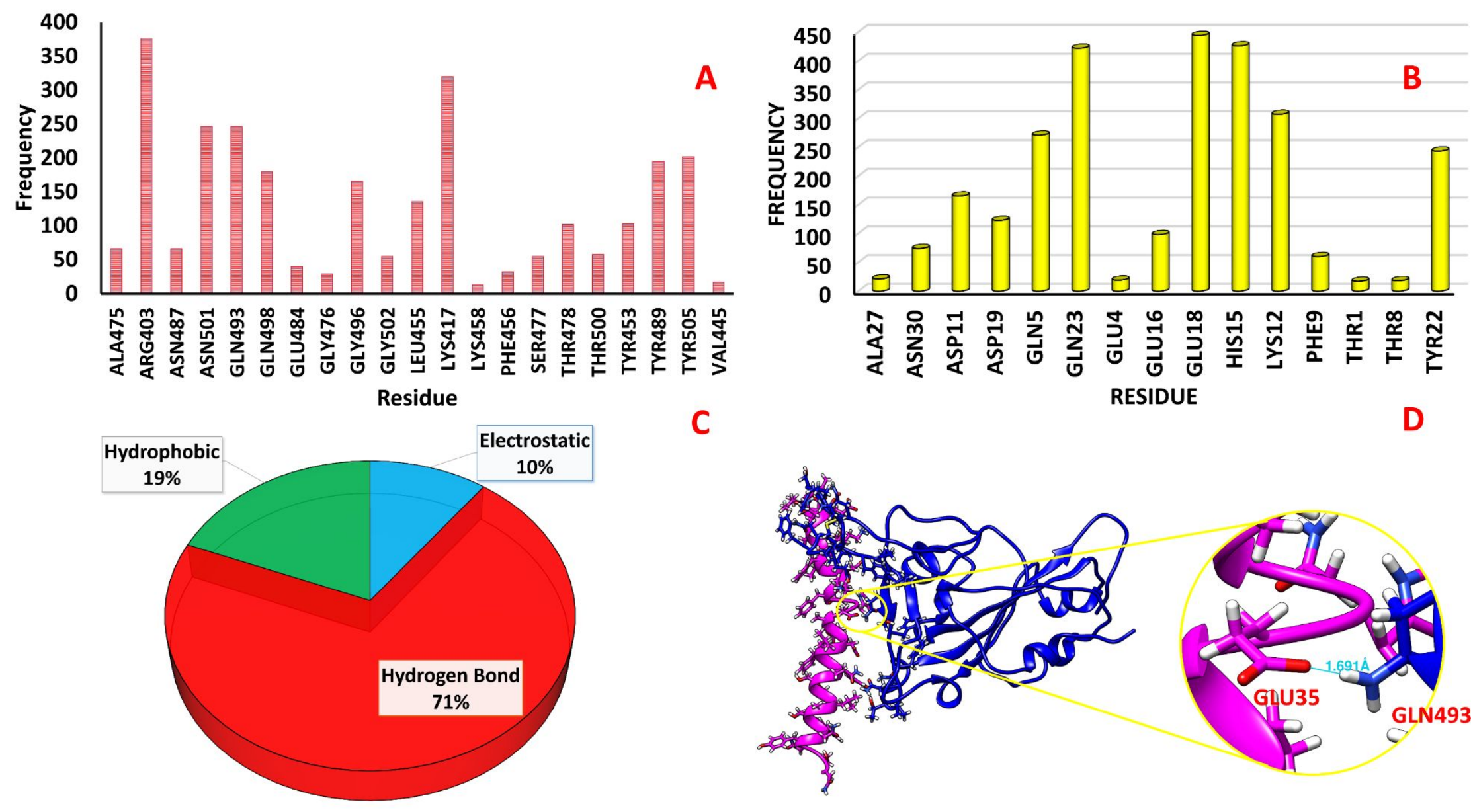

C

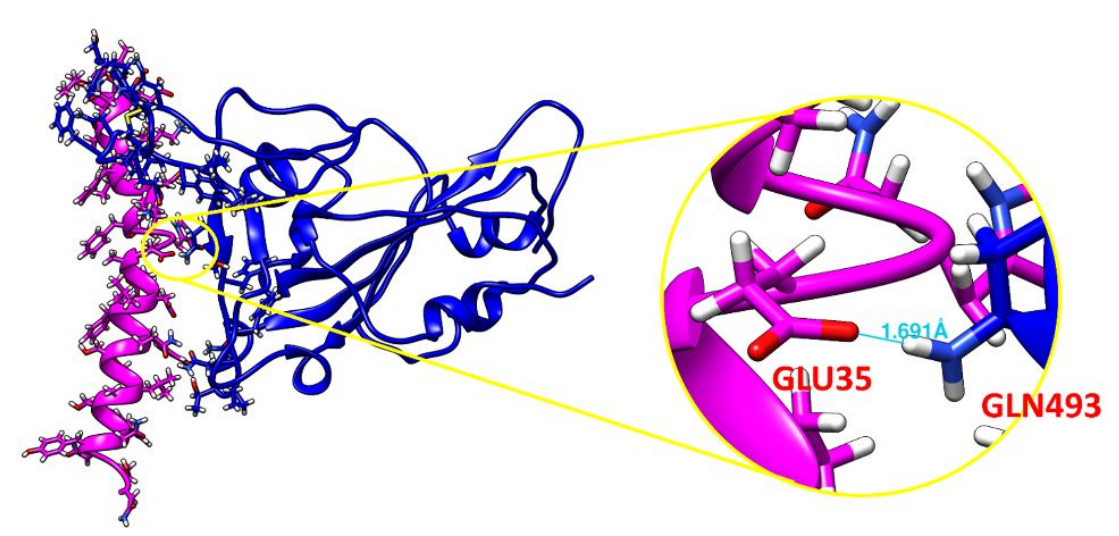

Figure S2. (a) Interacting residues in RBD; (b) Interacting residues in peptide AH; (c) Distribution of non-covalent interactions in AH-RBD complex obtained from 150 ns simulation time span; (d) A representative snapshot of AH-RBD complex highlighting interaction between AH (magenta) and RBD (blue). 